\title{
Circumambulatory Rearrangement with Characteristics of a 2:1 Covalent Molecular Bevel Gear
}

\author{
Rosa E. Bulo, Florian Allaart, Andreas W. Ehlers, Franciscus J. J. de Kanter, Marius Schakel, Martin \\ Lutz, Anthony L. Spek, and Koop Lammertsma
}

\begin{abstract}
Vrije Universiteit, FEW, Department of Chemistry, De Boelelaan 1083, 1081 HV Amsterdam, The Netherlands, Department of Chemistry and Applied Biosciences, ETH Zürich, USI-Campus, Via Giuseppe Buffi 13, 6900 Lugano, Switzerland, and Bijvoet Center for Biomolecular Research, Crystal and Structural Chemistry, Utrecht University, Padualaan 8, NL-3584 CH, Utrecht, The Netherlands.
\end{abstract}

\section{Complete Reference 22:}

Frisch, M. J.; Trucks, G. W.; Schlegel, H. B.; Scuseria, G. E.; Robb, M. A.; Cheeseman, J. R.; Zakrzewski, V. G.; Montgomery, Jr., J. A.; Stratmann, R. E.; Burant, J. C.; Dapprich, S.; Millam, J. M.; Daniels, A. D.; Kudin, K. N.; Strain, M. C.; Farkas, O.; Tomasi, J.; Barone, V.; Cossi, M.; Cammi, R.; Mennucci, B.; Pomelli, C.; Adamo, C.; Clifford, S.; Ochterski, J.; Petersson, G. A.; Ayala, P. Y.; Cui, Q.; Morokuma, K.; Malick, D. K.; Rabuck, A. D.; Raghavachari, K.; Foresman, J. B.; Cioslowski, J.; Ortiz, J. V.; Baboul, A. G.; Stefanov, B. B.; Liu, G.; Liashenko, A.; Piskorz, P.; Komaromi, I.; Gomperts, R.; Martin, R. L.; Fox, D. J.; Keith, T.; Al-Laham, M. A.; Peng, C. Y.; Nanayakkara, A.; Gonzalez, C.; Challacombe, M.; Gill, P. M. W.; Johnson, B.; Chen, W.; Wong, M. W.; Andres, J. L.; Gonzalez, C.; Head-Gordon, M.; Replogle, E. S. and Pople, J. A. Gaussian 98, Revision A.7, Gaussian, Inc., Pittsburgh PA, 1998.

\section{X-ray crystal structure determinations}

X-ray crystal structure determinations. X-ray intensities were measured on a Nonius Kappa CCD diffractometer with rotating anode (graphite monochromator, $\lambda=0.71073 \AA$ ). The structures were solved with automated Patterson methods (DIRDIF-99) ${ }^{30}$ and refined with SHELXL-97 ${ }^{31}$ against F2 of all reflections. Non hydrogen atoms were refined with anisotropic displacement parameters. All hydrogen atoms were located in the difference Fourier map. All hydrogen atoms in $\mathrm{a}-3$ and $\mathrm{H} 1$ and $\mathrm{H} 8$ in $\mathrm{a}-2$ were refined freely with isotropic displacement parameters. All other hydrogen atoms were refined with a riding model. Molecular illustration, structure checking and geometry calculations were performed with the PLATON package ${ }^{.32}$

a-2: C14H11O5PW, FW = 474.05, colourless needle, $0.57 \times 0.12 \mathrm{x}$ $0.06 \mathrm{~mm} 3$, orthorhombic, Pna21 (no. 33), $\mathrm{a}=18.9367(11), \mathrm{b}=$ 11.9364(5), c = 6.8799(4) $\AA, V=1555.11(14) \AA 3, Z=4, D x=2.025$ $\mathrm{g} / \mathrm{cm} 3, \mu=7.55 \mathrm{~mm}-1.39727$ Reflections were measured up to a resolution of $(\sin \theta / \lambda)_{\max }=0.65 \AA-1$ at a temperature of $110 \mathrm{~K}$. An absorption correction based on multiple measured reflections was applied (0.30-0.64 correction range). 3561 reflections were unique $($ Rint $=0.0378) .199$ parameters were refined with one restraint. $\mathrm{R} 1 / \mathrm{wR} 2 \quad[\mathrm{I}>2 \sigma(\mathrm{I})]: 0.0148 / 0.0252$. R1/wR2 [all refl.]: $0.0221 / 0.0269 . \mathrm{S}=1.065$. Flack parameter $\mathrm{x}=-0.006(6)$. Residual electron density between -0.87 and $0.36 \mathrm{e} / \AA^{3}$.

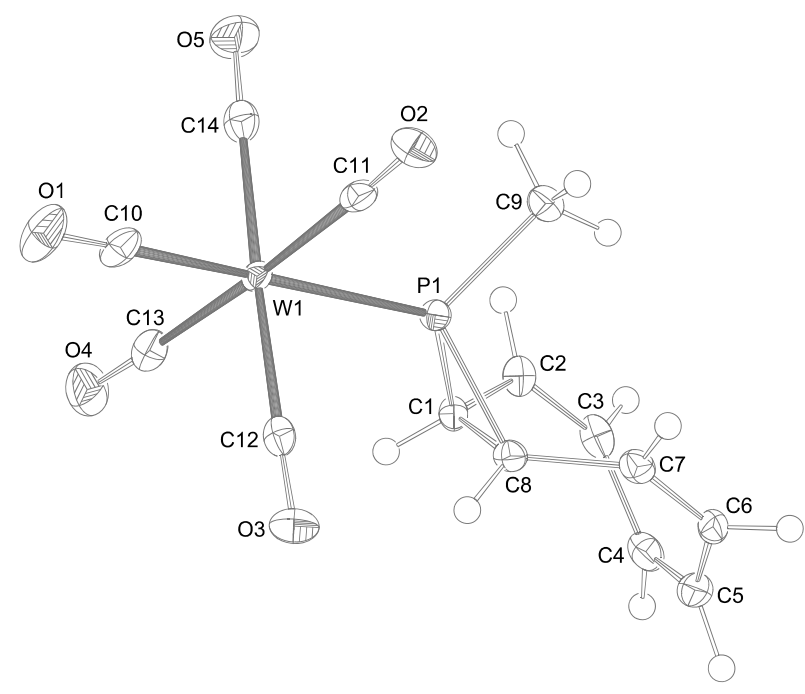

Figure S1. Displacement ellipsoid plot (50\% probability level) of a-2. Selected bond distances $(\AA)$, angles and torsion angles (deg.): P1-C1 1.832(3), P1-C8 1.838(3), C1-C2 1.490(4), C2-C3 1.319(4), C3-C4 1.465(4), C1-C8 1.525(5), C7-C8 1.490(4), P1-C9 1.816(3), P1-W1 2.4897(7); C1-P1-C8 49.11(12), C1-P1-C9 106.05(13), C8-P1-C9 106.52(13), C2-C1-C8 123.8(2), C1-C8-C7 125.2(2); C3-C2-C1-C8 55.8(4), C2-C3-C4-C5 -52.7(5), C4-C5-C6-C7 51.6(4), C6-C7-C8-C1 $-55.7(4)$.

a-3: C14H11O5PW, Fw = 474.05, yellow block, $0.36 \times 0.36 \times$ $0.30 \mathrm{~mm} 3$, monoclinic, $\mathrm{P} 21 / \mathrm{c}$ (no. 14), $\mathrm{a}=9.0373(11), \mathrm{b}=$ 13.4576(11), $c=13.5480(11) \AA, \beta=110.442(8)^{\circ}, \mathrm{V}=1543.9$ (3) $\AA 3$, $\mathrm{Z}=4, \mathrm{Dx}=2.039 \mathrm{~g} / \mathrm{cm} 3, \mu=7.60 \mathrm{~mm}-1.20279$ Reflections were measured up to a resolution of $(\sin \theta / \lambda) \max =0.65 \AA-1$ at a temperature of $150 \mathrm{~K}$. An analytical absorption correction was applied (0.17-0.37 correction range). 3522 reflections were unique $($ Rint $=0.0401)$. 234 parameters were refined with no restraints.

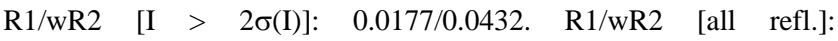
$0.0212 / 0.0448$. $\mathrm{S}=1.099$. Residual electron density between -1.08 and $0.61 \mathrm{e} / \AA ̊ 3$. 


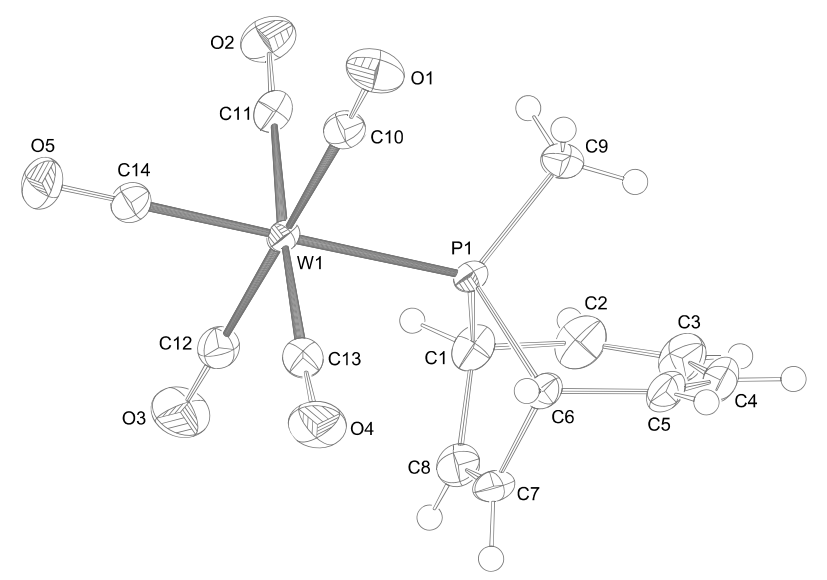

Figure S2. Displacement ellipsoid plot (50\% probability level) of $a-3$. Selected bond distances ( $\AA$ ), angles and torsion angles (deg.): P1-C1 1.850(3), P1-C6 1.846(3), C1-C2 1.512(4), C2-C3 1.341(5), C3-C4 1.455(5), C1-C8 1.521(5), C7-C8 1.320(5), P1-C9 1.804(3), P1-W1 2.5062(7); C1-P1-C6 90.85(14), C1-P1-C9 108.53(16), C6-P1-C9 108.40(15), C2-C1-C8 107.7(3), C5-C6-C7 109.4(2); C3-C2-C1-C8 -64.2(5), C2-C3-C4-C5 1.1(7), C4-C5-C6-C7 63.3(4), C6-C7-C8-C1 $-0.2(4)$.

CCDC 296483 (a-2) and 296482 (a-3) contain the supplementary crystallographic data for this paper. These data can be obtained free of charge from The Cambridge Crystallographic Data Centre via www.ccdc.cam.ac.uk/data request/cif.

\section{Calculated structures}

The Cartesian coordinates $(\AA)$ and total energies (a.u.) of the optimized structures using the (U)B3LYP exchange-correlation potentials with the 6-31G* basis set and LANL2DZ for chromium are given:

a2 $E($ tot $)=-1304.56106640$ a.u.

$\begin{array}{lrrr}\mathrm{P} & 0.33834800 & 0.53333400 & 0.00000000 \\ \mathrm{C} & -0.69984200 & 1.91344000 & 0.75300000 \\ \mathrm{C} & -0.69984200 & 1.91344000 & -0.75300000 \\ \mathrm{C} & -0.06975300 & 2.82414800 & 1.72500000 \\ \mathrm{C} & -0.06975300 & 2.82414800 & -1.72500000 \\ \mathrm{C} & 0.25915800 & 4.13522300 & 1.68500000 \\ \mathrm{C} & 0.25915800 & 4.13522300 & -1.68500000 \\ \mathrm{C} & 0.15766800 & 5.17007800 & 0.67900000 \\ \mathrm{C} & 0.15766800 & 5.17007800 & -0.67900000 \\ \mathrm{H} & 1.58820200 & 1.19073500 & 0.00000000 \\ \mathrm{H} & -1.61359800 & 1.46632300 & 1.15500000 \\ \mathrm{H} & -1.61359800 & 1.46632300 & -1.15500000 \\ \mathrm{H} & 0.08315200 & 2.33476800 & 2.68600000 \\ \mathrm{H} & 0.08315200 & 2.33476800 & -2.68600000 \\ \mathrm{H} & 0.62640400 & 4.52035300 & 2.63600000 \\ \mathrm{H} & 0.62640400 & 4.52035300 & -2.63600000 \\ \mathrm{H} & 0.15003200 & 6.16666800 & 1.12000000 \\ \mathrm{H} & 0.15003200 & 6.16666800 & -1.12000000 \\ \mathrm{CR} & 0.03656400 & -1.83850300 & 0.00000000 \\ \mathrm{C} & 0.03755400 & -1.83150200 & 1.90700000 \\ \mathrm{C} & 0.03755400 & -1.83150200 & -1.90700000 \\ \mathrm{C} & 1.93086800 & -2.04777500 & 0.00000000 \\ \mathrm{C} & -1.84962900 & -1.56682100 & 0.00000000 \\ \mathrm{C} & -0.19896700 & -3.69124500 & 0.00000000 \\ \mathrm{O} & 0.03336800 & -1.84090800 & 3.06000000 \\ \mathrm{O} & 0.03336800 & -1.84090800 & -3.06000000 \\ \mathrm{O} & 3.07645000 & -2.17332400 & 0.00000000 \\ \mathrm{O} & -2.99166900 & -1.40106600 & 0.00000000 \\ \mathrm{O} & -0.34451600 & -4.83685600 & 0.00000000\end{array}$

a2-tsIII $E($ tot $)=-1304.53874732$ a.u.

$\begin{array}{lrrr}\mathrm{P} & 0.00000000 & 0.59093600 & 0.00000000 \\ \mathrm{Cr} & 0.41893900 & -1.74148500 & 0.00000000 \\ \mathrm{H} & 0.94168600 & 1.63841000 & 0.00000000 \\ \mathrm{C} & -1.54915500 & 1.60664700 & 0.00000000 \\ \mathrm{H} & -2.39783900 & 0.91668200 & 0.00000000 \\ \mathrm{C} & -1.35801900 & 2.16949300 & 1.35336600 \\ \mathrm{C} & -1.35801900 & 2.16949300 & -1.35336600 \\ \mathrm{C} & -0.81763200 & 3.33922800 & 1.86051100 \\ \mathrm{C} & -0.81763200 & 3.33922800 & -1.86051100 \\ \mathrm{C} & -0.31275000 & 4.52276400 & 1.29388100 \\ \mathrm{C} & -0.31275000 & 4.52276400 & -1.29388100 \\ \mathrm{C} & -0.10795200 & 5.01046300 & 0.00000000 \\ \mathrm{H} & -1.74368600 & 1.48479200 & 2.10607000 \\ \mathrm{H} & -1.74368600 & 1.48479200 & -2.10607000 \\ \mathrm{H} & -0.78838900 & 3.34536100 & 2.94878900 \\ \mathrm{H} & -0.78838900 & 3.34536100 & -2.94878900 \\ \mathrm{H} & -0.00340800 & 5.23644600 & 2.05639600 \\ \mathrm{H} & -0.00340800 & 5.23644600 & -2.05639600 \\ \mathrm{H} & 0.31854100 & 6.01237100 & 0.00000000 \\ \mathrm{C} & 1.74520200 & -1.47546000 & 1.34910300 \\ \mathrm{C} & 1.74520200 & -1.47546000 & -1.34910300 \\ \mathrm{C} & -0.90938000 & -1.97909200 & 1.34663200 \\ \mathrm{C} & -0.90938000 & -1.97909200 & -1.34663200 \\ \mathrm{C} & 0.77456900 & -3.57822200 & 0.00000000 \\ \mathrm{O} & 2.54622800 & -1.32859000 & 2.16338000 \\ \mathrm{O} & 2.54622800 & -1.32859000 & -2.16338000 \\ \mathrm{O} & -1.71386200 & -2.12362200 & 2.15966200 \\ \mathrm{O} & -1.71386200 & -2.12362200 & -2.15966200 \\ \mathrm{O} & 0.99529500 & -4.71127300 & 0.00000000\end{array}$

a3 $E$ (tot) $=-1304.58753389$ a.u.

$\begin{array}{llrr}\mathrm{P} & 0.00000000 & 0.95059900 & 0.00000000 \\ \mathrm{H} & 1.02099800 & 1.92936900 & 0.00000000 \\ \mathrm{Cr} & 0.73726600 & -1.33940000 & 0.00000000 \\ \mathrm{C} & -1.16945700 & 1.57959200 & 1.33039000 \\ \mathrm{C} & -1.16945700 & 1.57959200 & -1.33039000 \\ \mathrm{C} & -2.51324100 & 1.31050200 & 0.66988400 \\ \mathrm{C} & -2.51324100 & 1.31050200 & -0.66988400 \\ \mathrm{C} & -1.05301900 & 3.06035200 & 1.62274400 \\ \mathrm{C} & -1.05301900 & 3.06035200 & -1.62274400 \\ \mathrm{C} & -0.99252800 & 4.07503000 & 0.73112900 \\ \mathrm{C} & -0.99252800 & 4.07503000 & -0.73112900 \\ \mathrm{H} & -1.03967000 & 1.00182100 & 2.24908200 \\ \mathrm{H} & -1.03967000 & 1.00182100 & -2.24908200 \\ \mathrm{H} & -3.41233600 & 1.22608400 & 1.27442900 \\ \mathrm{H} & -3.41233600 & 1.22608400 & -1.27442900 \\ \mathrm{H} & -1.04559300 & 3.32923000 & 2.67790700 \\ \mathrm{H} & -1.04559300 & 3.32923000 & -2.67790700 \\ \mathrm{H} & -0.93409300 & 5.07284500 & 1.16289900 \\ \mathrm{H} & -0.93409300 & 5.07284500 & -1.16289900 \\ \mathrm{C} & -0.53995300 & -1.77226400 & 1.33934600 \\ \mathrm{C} & -0.53995300 & -1.77226400 & -1.33934600 \\ \mathrm{C} & 1.99233700 & -0.86662400 & 1.34756500 \\ \mathrm{C} & 1.99233700 & -0.86662400 & -1.34756500 \\ \mathrm{C} & 1.37329800 & -3.09493000 & 0.00000000 \\ \mathrm{O} & -1.31274500 & -2.03409900 & 2.15570200 \\ \mathrm{O} & -1.31274500 & -2.03409900 & -2.15570200 \\ \mathrm{O} & 2.75417600 & -0.58640800 & 2.16800900 \\ \mathrm{O} & 2.75417600 & -0.58640800 & -2.16800900 \\ \mathrm{O} & 1.76945500 & -4.18050900 & 0.00000000\end{array}$

a3-tsI E(tot) = -1304.52387498 a.u.

$\begin{array}{lrrr}\mathrm{C} & 3.66855600 & 1.05435900 & 1.18131500 \\ \mathrm{C} & 4.10061400 & -0.27372700 & 1.28084500 \\ \mathrm{C} & 3.12118400 & 1.82970800 & 0.15466100 \\ \mathrm{C} & 3.98617200 & -1.41212500 & 0.42100500 \\ \mathrm{C} & 2.64846200 & 1.51973800 & -1.16176200 \\ \mathrm{P} & 0.62056800 & -0.73485400 & 0.39694100 \\ \mathrm{C} & 3.09611700 & -1.72206200 & -0.56083300 \\ \mathrm{C} & 2.15463700 & 0.37268100 & -1.70003300 \\ \mathrm{C} & 1.88452100 & -0.94450300 & -1.01288800 \\ \mathrm{H} & 3.90834700 & 1.64079500 & 2.06802100 \\ \mathrm{H} & 4.68287900 & -0.47372600 & 2.17908700 \\ \mathrm{H} & 3.10330000 & 2.89562300 & 0.37651400 \\ \mathrm{H} & 4.70519700 & -2.19981200 & 0.64827400 \\ \mathrm{H} & 2.62610800 & 2.38231700 & -1.82863700\end{array}$




$\begin{array}{lrrr}\mathrm{H} & 1.35715200 & -1.57635100 & -1.73795000 \\ \mathrm{H} & 0.76883000 & -2.01297300 & 0.99952200 \\ \mathrm{H} & 3.22477000 & -2.68582800 & -1.04902600 \\ \mathrm{H} & 1.85171600 & 0.41501800 & -2.74321800 \\ \mathrm{C} & -1.17560900 & 0.80771500 & -1.58908200 \\ \mathrm{Cr} & -1.60305500 & 0.02301800 & 0.09141800 \\ \mathrm{C} & -1.99938700 & -0.77206200 & 1.77765700 \\ \mathrm{C} & -3.42021600 & 0.44034700 & -0.15662100 \\ \mathrm{O} & -1.07782300 & 2.74240900 & 1.43718500 \\ \mathrm{C} & -1.26982800 & 1.72387500 & 0.94031100 \\ \mathrm{C} & -1.86287100 & -1.65786000 & -0.76773600 \\ \mathrm{O} & -2.24580500 & -1.25604600 & 2.79373000 \\ \mathrm{O} & -2.01002900 & -2.67277400 & -1.29581400 \\ \mathrm{O} & -0.96199800 & 1.28412200 & -2.61716900 \\ \mathrm{O} & -4.53654400 & 0.68838800 & -0.30565300\end{array}$

a3-tsII $E$ (tot) $=-1304.52210177$ a.u.

$\begin{array}{llll}\text { C } & -2.71701400 & 1.76868000 & 0.05191800\end{array}$

$\begin{array}{llll}\text { P } & -0.70256800 & -0.40463000 & -0.58685800\end{array}$

$\begin{array}{llll}\text { C } & -2.49118600 & 1.25257500 & 1.39158100\end{array}$

$\begin{array}{llll}\text { C } & -3.57200300 & 1.31295600 & -0.95490000\end{array}$

$\begin{array}{llll}\text { C } & -1.87552900 & -1.09790400 & 0.73568800\end{array}$

$\begin{array}{llll}\text { C } & -3.14337200 & -1.72708300 & 0.20226800\end{array}$

$\begin{array}{llll}\text { C } & -2.15562700 & -0.00364100 & 1.74065500\end{array}$

$\begin{array}{llll}\text { C } & -4.29638800 & 0.12577600 & -1.12169000\end{array}$

C $\quad-4.14034000-1.17252500 \quad-0.54929400$

$\begin{array}{llll}\mathrm{H} & -2.35583100 & 2.78626700 & -0.09476100\end{array}$

H $\quad-0.93953600 \quad-1.35399500 \quad-1.61837600$

$\begin{array}{llll}\mathrm{H} & -4.92969300 & -1.86864200 & -0.83178400\end{array}$

$\begin{array}{llll}\mathrm{H} & -2.53466200 & 2.00168200 & 2.18370100\end{array}$

$\begin{array}{llll}\mathrm{H} & -3.74846100 & 2.04851700 & -1.74017900\end{array}$

H $\quad-1.30889800 \quad-1.88797300 \quad 1.24180000$

$\begin{array}{llll}\text { H } & -3.26891200 & -2.77662200 & 0.46181500\end{array}$

$\begin{array}{llll}\mathrm{H} & -2.02504200 & -0.24724500 & 2.79190800\end{array}$

$\begin{array}{llll}\mathrm{H} & -5.07027400 & 0.17901400 & -1.88518100\end{array}$

$\begin{array}{llll}\mathrm{O} & 1.01870500 & 0.79855300 & 2.81756600\end{array}$

$\begin{array}{llll}\mathrm{Cr} & 1.60055300 & 0.01579300 & -0.08014800\end{array}$

$\begin{array}{llll}\text { C } & 3.43700900 & 0.15837900 & 0.26058400\end{array}$

$\begin{array}{llll}\text { C } & 1.96724800 & -0.47056600 & -1.88212000\end{array}$

$\begin{array}{llll}\text { C } & 1.20788900 & 0.50412000 & 1.71839400\end{array}$

$\begin{array}{llll}\text { C } & 1.62199900 & -1.81253700 & 0.44201400\end{array}$

$\begin{array}{llll}\text { C } & 1.47862800 & 1.86451500 & -0.59144300\end{array}$

$\begin{array}{llll}\mathrm{O} & 1.61383600 & -2.91901600 & 0.77187300\end{array}$

$\begin{array}{llll}\mathrm{O} & 2.19426900 & -0.76775200 & -2.97276800\end{array}$

$\begin{array}{llll}O & 4.57015800 & 0.23881300 & 0.46690000\end{array}$

$\begin{array}{llll}\text { O } & 1.40036500 & 2.97352100 & -0.88912900\end{array}$

s3 $E$ (tot) $=-1304.58709531$ a.u.

$\begin{array}{lrrr}\mathrm{P} & 0.00000000 & 1.26804700 & 0.00000000 \\ \mathrm{Cr} & -1.04929600 & -0.89024800 & 0.00000000 \\ \mathrm{H} & -0.92650900 & 2.34040000 & 0.00000000 \\ \mathrm{C} & 1.14487600 & 1.96343900 & 1.32521100 \\ \mathrm{C} & 1.14487600 & 1.96343900 & -1.32521100 \\ \mathrm{C} & 1.67852500 & 3.22478400 & 0.66983600 \\ \mathrm{C} & 1.67852500 & 3.22478400 & -0.66983600 \\ \mathrm{C} & 2.31470700 & 1.04979500 & 1.62465500 \\ \mathrm{C} & 2.31470700 & 1.04979500 & -1.62465500 \\ \mathrm{C} & 3.12872400 & 0.44270100 & 0.73105700 \\ \mathrm{C} & 3.12872400 & 0.44270100 & -0.73105700 \\ \mathrm{H} & 0.57961300 & 2.16564200 & 2.23996500 \\ \mathrm{H} & 0.57961300 & 2.16564200 & -2.23996500 \\ \mathrm{H} & 2.12239000 & 4.01179100 & 1.27441900 \\ \mathrm{H} & 2.12239000 & 4.01179100 & -1.27441900 \\ \mathrm{H} & 2.53264900 & 0.88953900 & 2.67873600 \\ \mathrm{H} & 2.53264900 & 0.88953900 & -2.67873600 \\ \mathrm{H} & 3.92728900 & -0.15870100 & 1.16192900 \\ \mathrm{H} & 3.92728900 & -0.15870100 & -1.16192900 \\ \mathrm{C} & 0.11959000 & -1.56094700 & 1.34816900 \\ \mathrm{C} & 0.11959000 & -1.56094700 & -1.34816900 \\ \mathrm{C} & -2.21126400 & -0.22481400 & 1.34640800 \\ \mathrm{C} & -2.21126400 & -0.22481400 & -1.34640800 \\ \mathrm{C} & -1.97278100 & -2.51195500 & 0.00000000 \\ \mathrm{O} & 0.79118500 & -2.00752400 & 2.17247100 \\ \mathrm{O} & 0.79118500 & -2.00752400 & -2.17247100 \\ \mathrm{O} & -2.92135400 & 0.17184300 & 2.16588800 \\ \mathrm{O} & -2.92135400 & 0.17184300 & -2.16588800 \\ \mathrm{O} & -2.54959400 & -3.51357200 & 0.00000000\end{array}$

\begin{tabular}{|c|c|c|c|}
\hline \multicolumn{4}{|c|}{ S2-ts $E($ tot $)=-130$} \\
\hline$P$ & & -0.39672600 & 0.00000000 \\
\hline $\mathrm{H}$ & -2.99362100 & -0.04671700 & 0.00000000 \\
\hline $\mathrm{Cr}$ & 0.00000000 & 1.39848900 & 0.00000000 \\
\hline C & -1.71253900 & -2.26446100 & 0.00000000 \\
\hline C & -1.16374700 & -2.50826300 & 1.35138400 \\
\hline C & -1.16374700 & -2.50826300 & -1.35138400 \\
\hline C & 0.08821300 & -2.79059400 & 1.86025100 \\
\hline C & 0.08821300 & -2.79059400 & -1.86025100 \\
\hline C & 1.34183000 & -3.09232500 & 1.29272800 \\
\hline C & 1.34183000 & -3.09232500 & -1.29272800 \\
\hline C & 1.85746800 & -3.21549100 & 0.00000000 \\
\hline $\mathrm{H}$ & -2.77610000 & -2.51770800 & 0.00000000 \\
\hline $\mathrm{H}$ & -1.94560900 & -2.41067800 & 2.10217500 \\
\hline $\mathrm{H}$ & -1.94560900 & -2.41067800 & -2.10217500 \\
\hline $\mathrm{H}$ & 0.10814900 & -2.79385800 & 2.94862700 \\
\hline $\mathrm{H}$ & 14900 & 385800 & 2700 \\
\hline $\mathrm{H}$ & 2.09647900 & -3.27685500 & 2.05580800 \\
\hline $\mathrm{H}$ & 2.09647900 & -3.27685500 & -2.05580800 \\
\hline $\mathrm{H}$ & 2.91571000 & -3.47124000 & 0.00000000 \\
\hline C & -1.07403300 & 2.20423 & 073600 \\
\hline C & -1.07403300 & 2.20423500 & -1.35073600 \\
\hline C & 1.10442300 & 0.61753700 & 1.35886900 \\
\hline C & 1.10442300 & 0.61753700 & -1.35886900 \\
\hline C & 1.09410200 & 2.90716000 & 0.00000000 \\
\hline $\mathrm{O}$ & -1.71033200 & 2.70958200 & 2.16871000 \\
\hline $\mathrm{O}$ & -1.71033200 & 2.70958200 & -2.16871000 \\
\hline $\mathrm{O}$ & 1.79657400 & 0.22112900 & 2.18875000 \\
\hline $\mathrm{O}$ & 1.79657400 & 0.22112900 & -2.18875000 \\
\hline $\mathrm{O}$ & 1.76873900 & 3.84548800 & 0.00000000 \\
\hline
\end{tabular}

(30) Beurskens, P. T.; Admiraal, G.; Beurskens, G.; Bosman, W. P.; Garcia-Granda, S.; Gould, R. O.; Smits, J. M. M.; Smykalla, C.; 1999, The DIRDIF99 program system, Technical Report of the Crystallography Laboratory, University of Nijmegen, The Netherlands.

(31) Sheldrick, G. M.; SHELXL-97, University of Göttingen, Göttingen (Germany), 1997.

(32) Spek, A. L.; J. Appl. Cryst. 2003, 36, 7-13. 